\title{
Retrospective Cross-sectional Study of Dengue Cases in IPD with Reference to Treatment- Monitoring \& Outcome in KEM Hospital, Mumbai
}

\author{
Anil Pardeshi ${ }^{1, *}$, Ratnendra Shinde ${ }^{2}$, Abhijeet Jagtap ${ }^{1}$, Ravindra Kembhavi ${ }^{2}$, Mayur Giri ${ }^{2}$, Snehal Kavathekar ${ }^{1}$ \\ ${ }^{1}$ P.G. scholars of School of Pharmaceutical-Medicine \\ ${ }^{2}$ Department of Community Medicine, Seth G. S. Medical College \& KEM Hospital, Parel, Mumbai \\ *Corresponding author: anil.pardeshi@cipla.com
}

Received June 23, 2014; Revised July 11, 2014; Accepted September 26, 2014

\begin{abstract}
Dengue is the most important vector-borne viral disease of humans and likely more important than malaria globally in terms of morbidity and economic impact [8]. Transmission of dengue is now present in every region of the world and more than 125 countries are known to be dengue endemic. Maharashtra has the highest dengue mortality rate (3.4\%) in India, as per data obtained from National Vector Borne Diseases Control Program (NVBDCP-2013). The case fatality rate is significantly high as compared that with other infectious diseases. This observational study was done to obtain the information on treatment given \& its outcome in clinical profile \& also to assess treatment gap \& outcome of Dengue patients admitted in KEM Hospital. The data was collected from the case records of dengue diagnosed patients from Medical record department and it was filled in case record form. The statistical-analysis \& the conclusion were drawn with the help of observations. The study revealed that maximum number (121) of dengue cases were in the age group of 21-30 yrs. There was an association between platelet count and treatment outcome. Severity in sign \& symptoms lead to complication $(\mathrm{n}=23)$ and death $(\mathrm{n}=21)$ in dengue cases. There were 22 deaths occurred in year 2013 because of dengue. Out of which maximum 12 cases reported in months of September $(n=6)$ \& October $(n=6)$. There was no treatment gap found in cases of Deaths in study but $36 \%$ of died patients received antimalarial treatment even after diagnosed with Dengue. Remaining 64\% of died patients received appropriate, rational and life saving treatment for dengue.
\end{abstract}

Keywords: dengue cases, treatment monitoring, treatment outcome, treatment gap

Cite This Article: Anil Pardeshi, Ratnendra Shinde, Abhijeet Jagtap, Ravindra Kembhavi, Mayur Giri, and Snehal Kavathekar, "Retrospective Cross-sectional Study of Dengue Cases in IPD with Reference to TreatmentMonitoring \& Outcome in KEM Hospital, Mumbai." American Journal of Epidemiology and Infectious Disease, vol. 2, no. 4 (2014): 97-100. doi: 10.12691/ajeid-2-4-3.

\section{Introduction}

Transmission of dengue is now present in every region of the world and more than 125 countries are known to be dengue endemic [5]. India has endemic for six major vector-borne diseases (VBD) namely malaria, dengue, chikungunya, filariasis, Japanese encephalitis and visceral leishmaniasis [1]. Estimates of the global incidence of dengue infections per year have ranged between 50 million and 200 million; however, recent estimates using cartographic approaches suggest this number is closer to almost 400 million [5]. Dengue causes more illness and death than any other arboviral infection [6].

Dengue afflicts all levels of society but the burden may be higher among poor peoples who live in communities [10]. WhileDengue fever/dengue hemorrhagic fever is now one of the most important public health problems in tropical developing countries and also has major economic and societal consequences.

There is no specific treatment for dengue other than symptomatic \& supportive measures with judicious fluid therapy. So, this observational study was done to obtain the information on treatment given \& its outcome in clinical profile $\&$ also to assess treatment gap regarding treatment outcome of Dengue patients admitted in KEM Hospital.

\section{Study Design \& Methodology}

This was a retrospective observational study. The study duration was 6 months aimed to study the review of Dengue cases in IPD of KEM Hospital, Parel, Mumbai, with respect to treatment monitoring \& outcome. The record of the patients admitted in IPD since last one year had been reviewed and observation was recorded and the study was reviewed by the Institutional Ethics Committee (IEC), Seth GS Medical College and KEM Hospital, Parel, Mumbai. The patients fulfilling the inclusion criteria i.e. the patients diagnosed with Dengue \& not the suspected cases were enrolled in the study. The number of dengue cases detected during 1 January 2013 to 31 December 2013 was considered as a sample size. In this study we 
collected the data of demography, clinical signs on admission, treatment and change in treatment on discharge, duration of treatment and Outcome of treatment. In this way it was assessed the whole profile of dengue patients and their treatment along with outcome. The case of dengue was defined as per WHO criteria. The data was collected from Medical record department and it was filled in case record form in paper system. The study was initiated only after getting the ethical approval from Ethics Committee, K.E.M Hospital, Mumbai. We have used descriptive statistics for calculation of quantitative \& qualitative variables. And chi-square test was used to find out the association between different demographic features \& outcome of dengue cases.

\section{Results}

In our study we reviewed 420 dengue patients' case papers. In total 420 patients, there were 236 (56\%) males \& 184 (44\%) females. Average age of Dengue patients was found to be 22 years \& 3 months. 130 (30.95\%) cases were reported from Paediatric ward while 290(69.05\%) cases reported from various Medicinal wards (Malemedical ward \& Female Medical ward) including Fever ward of KEM Hospital.

For age group less than 6 years, there were 45 cases, while age groups between 7-12, 13-20, 21-30, 31-40 \& more than 40 years had 85, 81, 121, 43 \& 45 Dengue cases respectively. Average stay of dengue patients in KEM Hospital was 4.54 days. 39\% $(n=162)$ of the patients had lowest IPD stay of $1-3$ days, while $16 \%(n=71)$ of the Dengue patients had longest IPD stay of 7-18 days. $61.42 \%(n=258)$ of the patients had mild sign \& symptoms of dengue while $13.8 \%(n=58)$ had severe sign \& symptoms. 26.43\% (111) cases had lowest platelet count i.e. below 60,000/cu-mm, whereas $19.76 \%(n=83)$ cases had count greater than 1, 80,000/cu-mm. 84.29\% $(n=354)$ cases had received electrolyte fluids either of DNS, RL, NS or combination of both of them. While $4.29 \% \quad(n=18)$ cases received blood components i.e. Platelet or FFP or WB. In parenteral drug therapy 83.10\% $(n=349)$ cases received symptomatic treatment while $5.95 \%(n=25)$ cases received life saving- emergency drugs. There were $5.24 \%(n=22)$ of the dengue patients leads to Death in the year of 2013. $0.95 \%(n=4)$ cases turned into complication, while $11.67 \%(n=49)$ cases recovered from complication. $80 \%(\mathrm{n}=336)$ cases completely recovered from dengue and remaining $2.14 \%(n=9)$ cases got discharge against medical advice (DAMA).

In the month of September \& October 2013, maximum number of 6 deaths in each month was reported. The Case fatality Rate of Dengue Cases in 2013 is found to be $11.67 \%$.

\section{Discussion}

The study done by Mena Lora AJ \& et.al, (Nov. 2013) was a cross-sectional retrospective review of patients with a clinical diagnosis of dengue pediatric patients in the Dominican Republic. They found that most important factors associated with severe dengue were rash, severe thrombocytopenia, and anemia $(\mathrm{P}<0.01)$ which are the same results of our study. Also these signs and symptoms were also associated with mortality.

A retrospective study was conducted by Aung KL \& et.al, (July 2013) reviewing medical records of 323 adult patients hospitalized with dengue infection, in order to determine factors associated with severe clinical manifestations of dengue. Using the revised 2009 WHO definitions, it was analyzed the results of dengue patients as $72.1 \%$ of the patients had non-severe dengue infection while in our study it was found to be $80 \%$ and also (27.9\%) of the patients had severe dengue infection in their study, while we found $(17.86 \%)$ of severe dengue cases. We had 9 (2.14\%) Dengue patients as DAMA.

The study undertaken by Sam SS \& et.al, (May 2013) was a retrospective study to examine dengue death cases in hospital from June 2006 to October 2007 with a view to determine if there had been changes in the presentation of severe to fatal dengue. Gastrointestinal pain, vomiting, diarrhea, intravascular leakages and bleeding occurred in the majority of cases. While in our study, we found that bleeding, diarrhoea, abdominal pains, shock, and hypotension were fatal signs of severe Dengue. It was concluded that in cohort, dengue fatalities were seen primarily in adult females with secondary dengue infection. While the study done by us reveals that, out of 22 dengue deaths, 13 (59.09\%) were males \& 9 (40.90\%) were females. The majority of the patients presented with common clinical and laboratory warning signs of severe dengue.

The study done by Alves JA \& et.al, (Dec.2011) aimed to characterize the epidemiological incidence of dengue in the period 2001-2010. This was an epidemiological study of dengue in the municipality of Aracaju, state of Sergipe, in the period between 2001 and 2010,A descriptive analysis of the number of confirmed cases of dengue, according to year, semester, sanitary district, age, and sex, was performed. It was observed \& further concluded that in 2008, a significant increase in the proportion of dengue cases compared with other previous years was verified. While, in our study ... Data were collected from Jan.2013 Dec.2013, \& it was found that in October month out of 420 total dengue cases , 123 (29.29\%) i.e. the largest Dengue cases were found \& lowest i.e.1 case of dengue was found in February month of 2013.In September month also, larger dengue cases i.e. 121 out of 420 (28.81\% ) were found. This indicates that only September \& October month of 2013, 58.10\% (244 out of 420) dengue cases were found. It was concluded that climate affects mainly the range of vector borne diseases, whereas weather affects the timing and intensity of outbreaks [2].

\section{Conclusion}

There were 22 deaths occurred in year 2013 because of dengue. Out of which maximum 12 cases reported in months September $(n=6)$ \& October $(n=6)$. This indicates that season is also the main factor for this Vector borne disease. There was no treatment gap found in cases of Deaths in study but $36 \%$ of died patients received antimalarial treatment even after diagnosed with Dengue. This may indicate that antimalarial treatment in these 34\% patients leads to resistance for the treatment. Remaining 
$64 \%$ of died patients received appropriate, rational and life saving treatment for dengue.

The severity of sign \& symptoms i.e. Hypotension, bleeding, shock and multi-organ failure leads to mortality. Death of 22 dengue patients and complication in 4 patients is a serious issue regarding to health care. So we suggest that may be there is need of early diagnosis of dengue cases \& that will be minimizes the further incidence of morbidity \& mortality of dengue cases.

Limitation of study- As this was retrospective study, so we depended on secondary data. Prospective study should be needed to overcome this limitation. We reviewed Dengue cases only from KEM Hospital, multicentric study should be done.

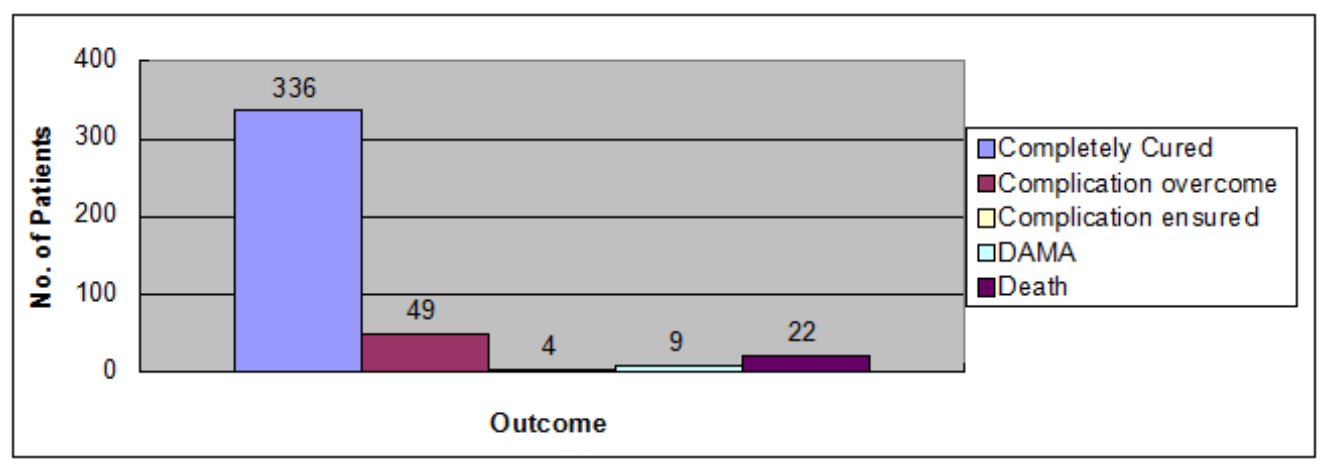

Figure 1. No. of Dengue patients \& Treatment outcome

Table 1. Sex wise distribution of Dengue cases

\begin{tabular}{|c|c|c|}
\hline Sr. no. & Sex & No. Of cases \\
\hline 1. & Male & $236(56 \%)$ \\
\hline 2. & Female & $184(44 \%)$ \\
\hline & Total & 420 \\
\hline
\end{tabular}

Table 2. No. of cases with age groups

\begin{tabular}{|c|c|c|c|}
\hline Sr. no. & Age category & No. of cases & Percentage \\
\hline 1. & $0-6$ & 45 & $10.71 \%$ \\
\hline 2. & 7 to12 & 85 & $20.24 \%$ \\
\hline 3. & $13-20$ & 81 & $19.29 \%$ \\
\hline 4. & $21-30$ & 121 & $28.81 \%$ \\
\hline 5. & $31-40$ & 43 & $10.24 \%$ \\
\hline 6. & $>40$ & 45 & $10.71 \%$ \\
\hline
\end{tabular}

Table 3. fluid therapy in dengue cases

\begin{tabular}{|c|c|c|c|}
\hline Sr. no. & Fluid therapy & No. of cases & percentage \\
\hline 1. & Electrolytes & 354 & $84.29 \%$ \\
\hline 2. & Not given & 48 & $11.43 \%$ \\
\hline 3. & Blood components & 18 & $4.29 \%$ \\
\hline
\end{tabular}

Table 4. Treatment Outcome

\begin{tabular}{|c|c|c|c|}
\hline Sr. no. & Treatment outcome & No. Of cases & percentage \\
\hline 1. & Completely Cured without complication & 336 & $80.00 \%$ \\
\hline 2. & With Complication but cured & 49 & $11.67 \%$ \\
\hline 3. & required referral elsewhere & 4 & $0.95 \%$ \\
\hline 4. & DAMA & 9 & $2.14 \%$ \\
\hline 5. & Death & 22 & $5.24 \%$ \\
\hline & Total & 420 & \\
\hline
\end{tabular}

Table 5. Duration of stay in hospital of dengue cases \begin{tabular}{|l|l|l|l|}
\hline Sr. no. & Duration of stay in hospital in days & No. Of cases & Percentage \\
\hline
\end{tabular}

\begin{tabular}{|c|c|c|c|}
\hline 1. & 1 to 3 & 162 & $39 \%$ \\
\hline 2. & 4 to 6 & 187 & $45 \%$ \\
\hline 3. & 7 to 18 & 71 & $16 \%$ \\
\hline
\end{tabular}

\section{References}

[1] Agarwal, R, Kapoor, S, Nagar, R, \& et al. "A clinical study of the patients with dengue hemorrhagic fever during the epidemic of 1996 at Lucknow, India." Southeast Asian J Trop Med Public Health; 30: 735-40. 1999.

[2] Ahmed, MM and et al, "Clinical profile of dengue fever infection in King Abdul Aziz University Hospital Saudi Arabia.” J Infect Dev Ctries 3; 4 (8): 503-10, Sept. 2010.
[3] Alves, JA, Santos, JR, Mendonca, EN, "Epidemiological aspects of dengue in Aracaju, State of Sergipe, Brazil.” Rev Soc Bras Med Trop. 44 (6):670-3, Nov-Dec 2011.

[4] Anderson, CR, Downs, WG, \& Hill, AE. "Isolation of dengue virus from a human being in Trinidad. Science.” 124 (3214): 2245,1956. [PubMed]

[5] Arboleda, M, Campuzano, M, Restrepo, BN, \& et.al, "The clinical behavior of dengue in patients hospitalized in the Antonio Roldán Betancur Hospital of Apartadó, Antioquia, 2000.” Emerg Infect Dis. 13 (6):924-5, June2007.

[6] Aung, KL, Thanachartwet, V, \& Desakorn, V, "Factors associated with severe clinical manifestation of dengue among adults in Thailand.” Biomedica ; 26 (2): 286-94. Jun. 2006.

[7] Balmaseda, A, Hammond, SN, Tellez, Y, Imhoff, L, Rodriguez, Y, Saborio, SI, \& et al. "High seroprevalence of antibodies against dengue virus in a prospective study of schoolchildren in Managua,Nicaragua.” Trop Med. Int Health; 11: 935-42. 10. 2006 [PubMed]

[8] Bhattacharjee, N, Mukherjee, KK, Chakravarti, SK, Mukherjee, $\mathrm{MK}$, \& et al. "Dengue haemorrhagic fever (DHF) outbreak in Calcutta-1990.” J Commun Dis.; 25: 10-4, 1993.

[9] Brasil. Ministério da Saúde. Secretaria de Vigilância em Saúde Dengue: diagnóstico e manejo clínico. 2nd ed Brasília, DF; 2005.

[10] Carey, DE, Myers, RM, Reuben, R, \& Rodrigues, FM, "Studies on dengue in Vellore, South India.” Am J Trop Med Hyg.; 15: 580-7, 1966.

[11] Chambers, TJ, Hahn, CS, Galler, R, \& Rice, CM. "Flavivirus genome organization, expression, and replication.” Annu Rev Microbiol.; 44: 649-88, 1990. [PubMed]

[12] Chatterjee, SN, Chakravarti, SK, Mitra, AC, \& Sarkar, JK, "Virological investigation of cases with neurological complications during the outbreak of haemorrhagic fever in Calcutta.” J Indian Med Assoc.; 45: 314-6, 1965.

[13] Cherian, T, Ponnuraj, E, Kuruvilla, T, \& et.al, “An epidemic of dengue haemorrhagic fever \& dengue shock syndrome in \& around Vellore.” Indian J Med Res.; 100: 51-6, 1994.

[14] Chuang, V, Wong, TY, Leung, YH, "Review of dengue fever cases in Hong Kong during 1998 to 2005.” Hong Kong Med J.; 14 (3): 170-7, Jun.2008.

[15] Dar, L, Broor, S, Sengupta, S, Xess, I, \& Seth, P, "The first major outbreak of dengue hemorrhagic fever in Delhi, India.” Emerg Infect Dis.; 5: 589-90, 1999.

[16] Faddy, HM, Seed, CR, Fryk, JJ, "Implications of dengue outbreaks for bloodsupply, Australia .”Emerg.Infect.Dis.; 19 (5): 787-9, May. 2013.

[17] Gunasekaran, P, Kaveri, K, Mohan, S. "Dengue disease status in Chennai (2006-2008): a retrospective analysis.” Indian J Med Res.; 133: 322-5, Mar. 2011.

[18] Kabra, SK, Verma, IC, Arora, NK, \& et.al, "Dengue haemorrhagic fever in children in Delhi.” Bull World Health Organ.; 70:105-8, 1992. 
[19] Katherine, L. Anders, \& et.al, "Epidemiological factors associated with DSS \& mortality in hospitalized Dengue patients in Vietnam.” Am J Trop Med Hyg. 5; 84 (1): 127-134. January 2011.

[20] Khursheed, M, Khan, UR, \& Ejaz, K, “A comparison of WHO guidelines issued in 1997 and 2009 for dengue fever-single centre experience.” J Pak Med Assoc.; 63 (6): 770-1, Jun. 2013.
[21] Kimura, R, \& Hotta, S, "Studies on dengue fever (IV) on inoculation of dengue virus into mice.” Nippon Igaku.; 3379: 62933, 1944. 